\title{
The infinitely small: A mission, a passion, a vision for the 21st century
}

A s we celebrate the 25th anniversary of the Infectious Diseases Research Centre of Laval University, infections kill 17 million people a year and are the primary cause of mortality on this planet. Since 1980 , the mortality from infectious diseases has increased by $50 \%$ in North America, and 30 new infectious diseases and disease causing organisms (eg, Cryptosporidium parvum, Escherichia coli 0157/47, Helicobacter pylori, human immunodeficiency virus [HIV], human herpes virus [HHV]6 and HHV8, to name a few) have emerged. Micro-organisms are not only causing diseases, but their role in some forms of cancer, including $H$ pylori and stomach cancer, human papillomaviruses types 16 and 18 , and cervical cancer is now well established. The World Health Organization has estimated that over 1.5 million of the 10 million new cancer cases a year could be avoided by preventing the infections associated with them. Infectious diseases in North America are also a cause of considerable morbidity with important human, social and economic impact. Urinary tract infections alone are responsible for more than six million medical visits a year in the United States and Canada, and cause an astronomical number of lost days of work. Nosocomial infections, which affect two million people a year in both the United States and Canada, induce annual health care costs of $\$ 5$ billion.

The discovery and use of antibiotics has been one of the great achievements of humanity, but their overuse in humans, animals and even plants is disturbing the microbiological ecology. Twenty-five years ago, Haemophilus influenzae was starting to develop resistance, and in South Africa Streptococcus pneumoniae was already resistant to penicillin. The pharmacological industry had already conquered penicillinresistant staphylococci by elucidation of the beta-lactam ring by Rolinson and Sutherland in 1958; broad-spectrum cephalosporins and aminoglycosides had arrived in the 1960s, and new classes of antimicrobials were rapidly being developed in the early 1970s; there was no doubt in the minds of scientists and clinicians in 1974 that infections could be overcome. In fact, the Surgeon General of the United States had said a few years before that we had already won the war against microbes.

When I arrived in Canada in 1974, infectious diseases were in their infancy, and a few pioneers such as Allan Ronald, George Goldsand, Hugh Robson and André Marcoux, to name a few, had already started their infectious diseases training programs. The prospect of curing diseases was appealing to the young clinicians and scientists specializing in infectious diseases at that time, who are now the Canadian leaders in the field of infectious diseases. This wind of positivism, driven by the idea that new broad-spectrum antibiotics could control any bacteria, lasted throughout the 1970s and a good part of the early 1980s. During that euphoric period, the Canadian Infectious Diseases Society was founded. Our specialty was approved by the Royal College of Physicians and Surgeons of Canada, and we were contemplating the creation of The Canadian Journal of Infectious Diseases.

Those years were the apogee of the 'drug-bug' era, and during this short period we investigated more than 35 new antibiotics at either prephase I (in vitro animal models) or phases I, II and III at Laval University. Pyelonephritis, renal toxicity of aminoglycosides, pharmacology of antimicrobials and their tissue distribution were the initial foci of our research endeavours.

By 1983, when Luc Montagnier discovered HIV, AIDS was increasing at an alarming rate. Canadian infectious disease specialists, as well as others in the world, had to face for the first time daily deaths of patients; many of our colleagues had difficulty coping with this new paradigm. In the following years, industry that had saturated the antibiotic market started to devote their efforts toward antiviral research to such an extent that they neglected bacterial diseases and the emergence of antibiotic resistance in Gram-positive bacteria that we now face.

AIDS and antiviral research became an instant priority for the researchers at the Infectious Diseases Research Centre at Laval University. AIDS gave us the opportunity to develop a new concept of global health research, where basic scientists, clinicians, epidemiologists, nurses and researchers in psychosocial services and public health would work under the same roof to conquer this disease. We are now the AIDS centre for the eastern part of the Province of Québec.

In the mid-1980s, it became clear to us that to improve prevention and control of infectious diseases and of the spread of antimicrobial resistance, research efforts must be directed toward a better understanding of microbial genomics and microbial pathogenesis. Our recruitment was, thus, directed toward those two objectives. We also tried to reach a balance between clinical and basic scientists, which, in my mind, is the direction medical research should take. A better understanding of the microbial genome, virulence factors and host responses has led our group to develop microbicides, vaccines against the meningococci, new immunoliposomes targeting HIV and rapid DNA-based diagnostic tests that provide results in less than $1 \mathrm{~h}$. I strongly believe that many microbiology laboratories in Canada would not have been centralized if microbiologists had had the tools with which to identify pathogens rapidly, providing physicians with the identity of the pathogens involved and their antibiotic resistance patterns, at the same time as biochemistry, hematology and radiology results are available. 
INFECTIOUS DISEASES RESEARCH TEAMS AT THE CRI

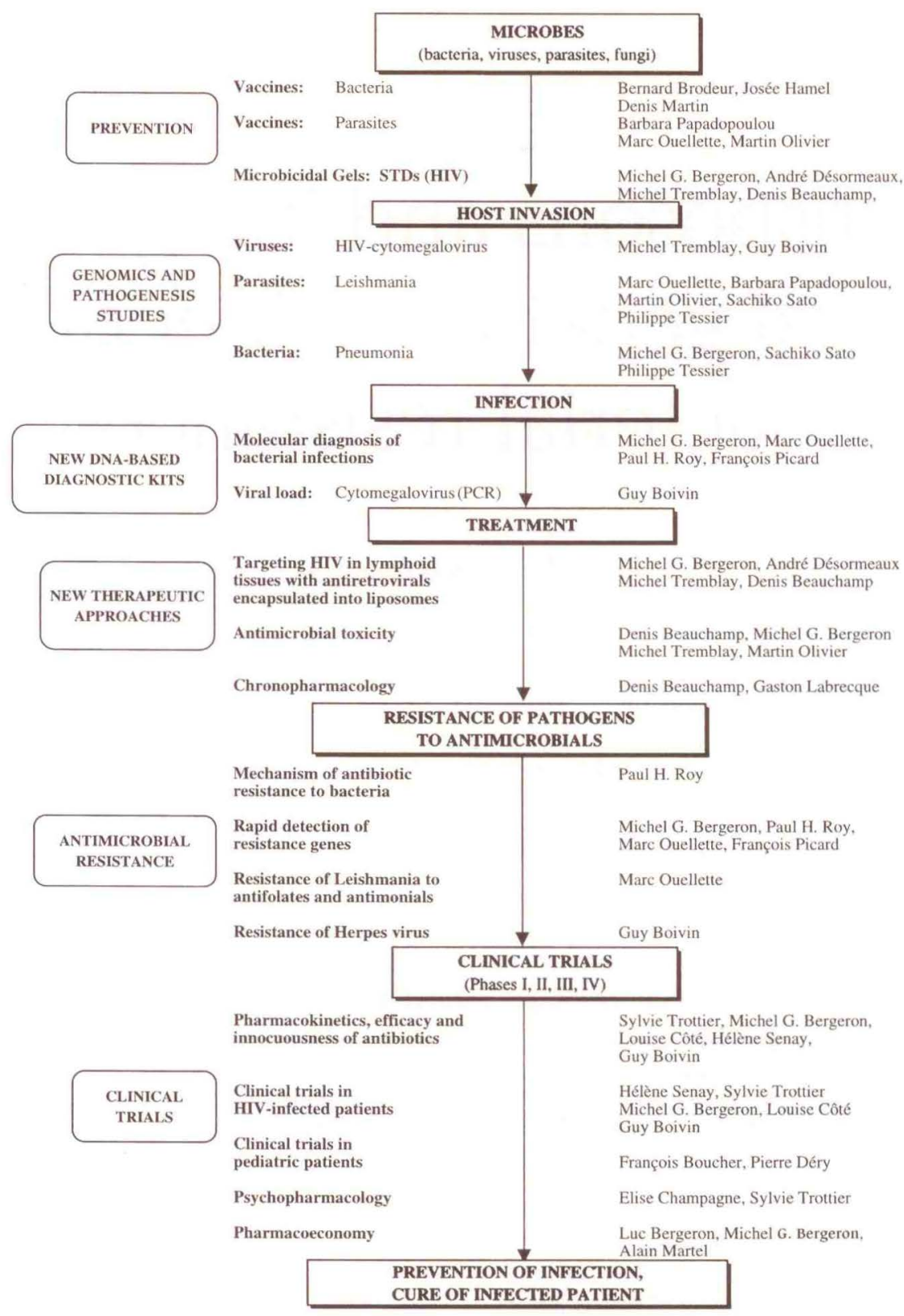

This chart summarizes the major research teams at the CRI and the names of the researchers working on these different research areas, which go from the genome to the patient. HIV Human immunodeficiency virus; STD Sexually transmitted disease; PCR Polymerase chain reaction

In 1974 the Centre de Recherche en Infectologie (CRI) was composed of only a few people, and I had an $\$ 11,000$ Medical Research Council of Canada grant. The CRI has grown to become an 'institute' where integrative research is a daily reality. One hundred and ninety people (20 independent researchers 12 basic scientists and eight clinical scientists - research associates and assistants, research nurses and supporting staff, and 72 graduate and postgraduate students and fellows) generated \$9 million in 1998 in grants, scholarships and contracts. We expect to reach $\$ 10$ million in 1999.

In this special supplement to The Canadian Journal of Infectious Diseases, we have highlighted some of the major research areas of the CRI of Laval University. Microbes, the first form of life, are 8.3 billion years old, and their genetic material can adapt to any situation. We always talk about the war against microbes - it is time we learn more about microbes, not to kill them, but to control them, while respecting the microbial ecosystem of which we as humans are an integral part.

Michel G Bergeron MD FRCPC Professor, Chairman and founder of the Centre de Recherche en Infectiologie, Université Laval, Québec City 


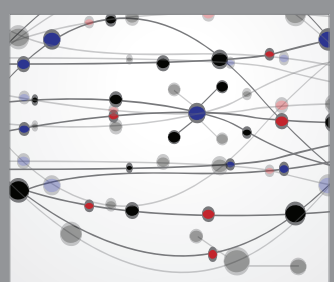

The Scientific World Journal
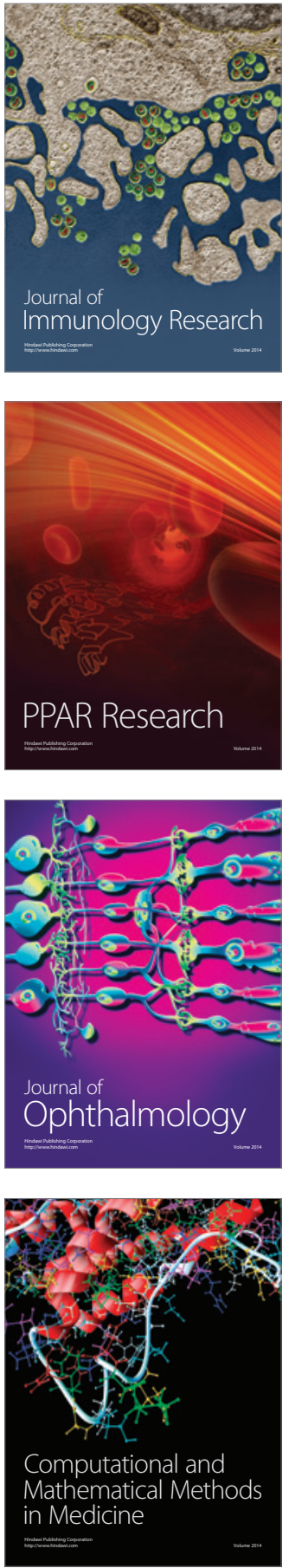

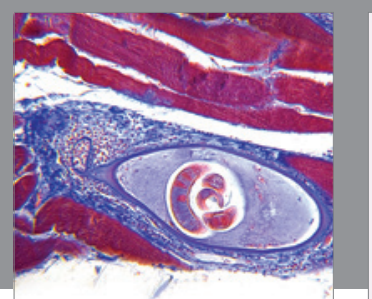

Gastroenterology Research and Practice

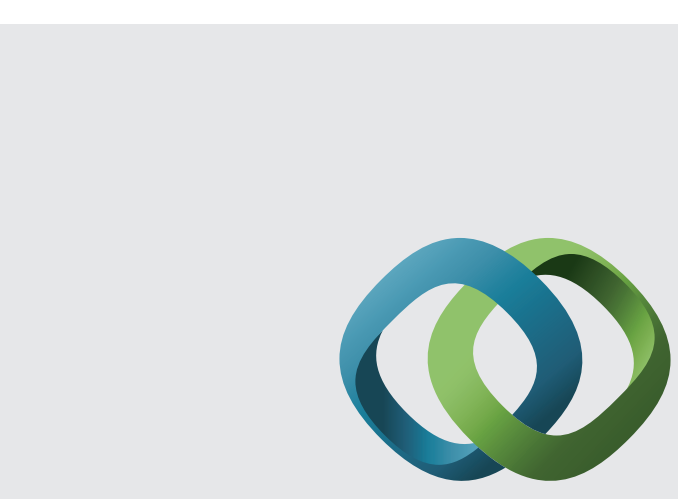

\section{Hindawi}

Submit your manuscripts at

http://www.hindawi.com
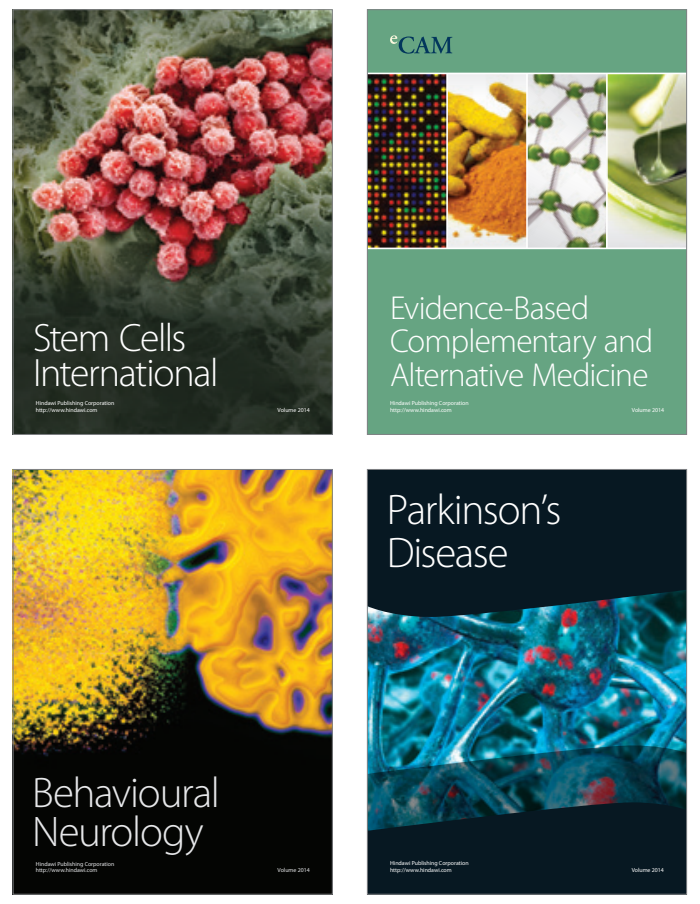
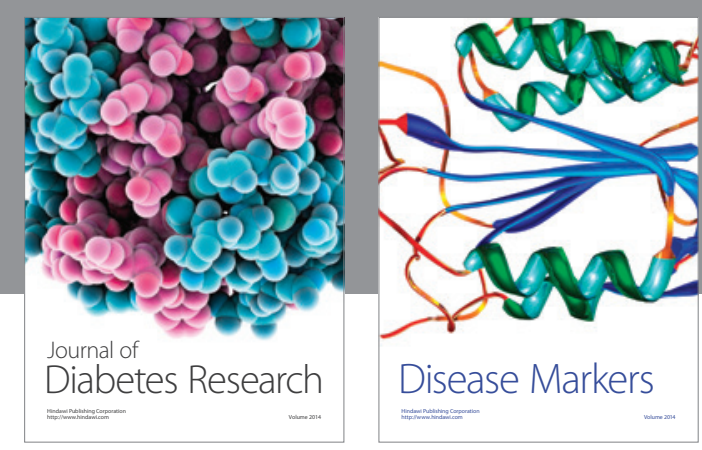

Disease Markers
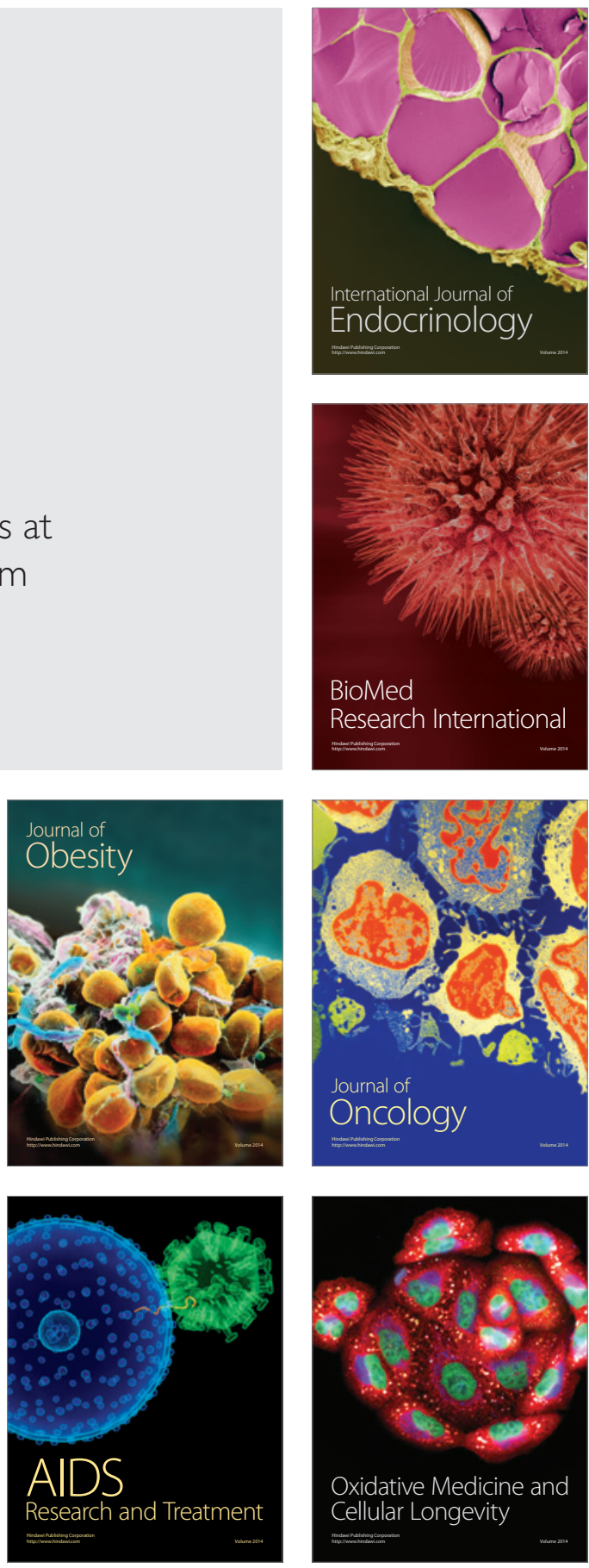